\title{
Health Systems as Complex Systems
}

\author{
Mireya Martínez-García ${ }^{1}$, Enrique Hernández-Lemus ${ }^{2,3^{*}}$ \\ ${ }^{1}$ Graduate Program in Health System’s Management, National Autonomous University of México, México DF, Mexico \\ ${ }^{2}$ Computational Genomics Department, National Institute of Genomic Medicine, México DF, México \\ ${ }^{3}$ Complexity in Systems Biology, Center for Complexity Sciences, National Autonomous University of México, México DF, México \\ Email: *ehernandez@inmegen.gob.mx
}

Received December 11, 2012; revised January 10, 2013; accepted January 22, 2013

\begin{abstract}
Health systems are paradigmatic examples of human organizations that blend a multitude of different professional and disciplinary features within a critically performance environment. Communication failure and defective processes in health systems have a tremendous impact in society, both in the financial and human aspects. Traditionally, health systems have been regarded as linear hierarchic structures. However, recent developments in the sciences of complexity point out to health systems as complex entities governed by non-linear interaction laws, self-organization and emergent phenomena. In this work we review some aspects of complexity behind health systems and how they can be applied to improve the performance of healthcare organizations.
\end{abstract}

Keywords: Health Systems; Complexity; Self-organization

\section{Introduction}

It is a proven fact that in all different levels and disciplines (from basic biomedicine to public health to healthcentered businesses) health care is becoming progressively more and more complex. For instance, few decades ago, the typical general practitioner worked under a set of individual premises (often dictated by his/her own wealth of experience), "...surrounded by a minimum of support staff, subscribed to a single journal, phoned up a specialist whenever he or she needed advice, and did around an hour's paperwork per week. The specialist worked in a hospital, focused explicitly on a particular system of the body, was undisputed leader of his or her firm, and generally left administration to the administrators. These individuals often worked long hours, but most of their problems could be described in biomedical terms and tackled using the knowledge and skills they had acquired at medical school..." [1]. Of course, in most instances, healthcare professionals these days face a much more entrenched environment in their day-to-day labor and public health policy delegates have to plan for highly complex scenarios of action [2,3].

As it is well known, there is no consensus definition of what is complexity. However, we may define complexity in an operational way by enumerating some issues characteristic of complex phenomena. Complex behavior emerge due to different kinds of interactions among con-

${ }^{*}$ Corresponding author. stituents in a system. By studying the relationship between different types of interactions, we may be able to disentangle, and predict, but also - to a certain extent- to manage and control the outcome of complex systems functioning. In brief, complex processes often show unpredictable behavior since synergistic effects emerge by the nonlinear combination of multiple inputs in a system. This is specially true in systems composed by autonomous constituents (also called agents) since these do not obey any global lock-and-trigger conditions. A second feature of complex systems (related to the previous one) is the presence of multiple causality, in contrast, one important characteristic of simple reductionistic systems is that $a$ single cause generates a single effect. This is not the case in complex systems for which a pleiad of inputs are confluent in a single output.

Societies are paradigmatic examples of complex behavior in this regard, and organizations (such as health systems for instance) are no exception: in order to get a desired result, a multitude of elements should do their $j o b$ so that everything fits well in such organization. Recognizing this fact is, of course, behind management and logistics strategies, and it is in fact, the basis for Operations Research. Multiple causality is often reinforced by phenomena related to circular causality, i.e. "effects" are fed back to modify "causes" and information flow amongst different hierarchical levels on the system [4]. Circular casuality is rooted in the presence of feedback, strange loops and complicated recursion patterns that may induce logical paradoxes. 
We may define feedback as a process in which a part of the output signal (i.e. the response) of a system is passed-out (fed-back) to the input (i.e. the "cause") thus leading to a loop (or recursive relation) in the functioning mechanisms of the system. There are two basic feedback schemes: positive feedback that increases the dynamics (i.e. increases the deviation of the system to an initial state, thus causing destabilization of the system) and negative feedback that does the opposite. The ideas behind positive and negative feedback are pretty old; we could traced them back to the notions of vicious circles and virtuous circles [5]. Primary models of feedback (both positive and negative) have been used for decades in engineering control science to stabilize the operation of production plants.

Other instances in which feedback phenomena lead to stability is public policy: periods of stability of governmental policies with occasional (and often abrupt) changes may be explained by the presence of positive and negative feedback processes [6]. Negative feedback processes lead to the stability of policies and institutions, while positive feedback results in self-amplification of trends and diffusion of new ideas, refreshing the socalled policy portfolio. Feedback processes in social dynamics have led to the construction od cooperativity structures such as the famous go-with-the winner strategy $[7,8]$ for which Thomas Schelling received the Nobel Prize in Economics (2005): individual decisions within a group are often determined by the behavior of others in the group: we are ready to cross the street with a red light-we shouldn't-, if others around us do the same. Complex feedback structures are rooted behind the notions of leadership, cooperativity, economic and political stability and even fashion trends. This may lead us to recognize the fact that collective phenomena often involves certain forms of self-organization. When wellunderstood, recognizing self-organizing patterns may lead to better control of complex systems dynamics. For instance, in modern societies (e.g. markets) not every action of an autonomous agent is externally regulated (say by the state). Internal regulatory mechanisms play a determinant role in systems dynamics, as has been recognized since the days of Adam Smith and his invisible hands theory of the markets.

Self-organization and circular causality schemes may induce another feature of complex systems, namely asymmetric statistics (or inequality, or non-equilibrium phenomena, or Paretto scaling). Asymmetric statistics arise from the fact that not every agent contributes (or receives "shares") in the same proportion to the functioning of a complex system (in the way Gaussian variables contribute to an average property in simple systems). This causes that asymmetric complex systems behavior is often described by skew power law probability distribu- tions. The paradigmatic example of such complex behavior is wealth asymmetry in economics as described by Paretto statistics: about $80 \%$ of the income is made by $20 \%$ of the people. Similar patterns have been observed not only in economy but also in biology (a small number of molecules is involved in the vast majority of metabolic reactions), in geophysics (everyday a great number of "little-earthquakes" happen everywhere in the world. However, the vast majority of geologic energy is released in a few very strong earthquakes focused in a small number of epicenters), in the architecture and dynamics of the world wide web (some few pages say www.google.com present the vast majority of links and most of the visits), and in namely every other complex system we may have in mind.

Complexity often arise (and this is central to the rest of this paper) from the structure of a system. It is known that there are selective and functional advantages to systems composed by hierarchically organized structures (i.e. systems composed of subsystems, again composed of sub-subsystems). This advantages will be related (as we may see later) with complex systems features such as modularity and robustness as already outlined in the pioneering works of Herbert Simon [9]. Of course, not every hierarchic strategy translates into an efficient functional feature, but recognizing that the different constituents of a system affect its behavior in a different manner is essential to understand complexity.

An important set of mathematical techniques that allow us to deal with non-trivial hierarchy relationships and structural complexity by representing the structural relationship among the elements in a complex systems is graph theory or, as is now called complex network theory (CNT). CNT offers a proper (some say even natural) way to represent systems with individual nodes as constituents or agents, and connections (or links or edges) playing the role of interactions or relationships. However, it is important to have in mind that structural complexity is just an element of complex behavior. For instance, even systems with relatively simple structure may lead to complicated dynamics [10]. The origin of complex behavior is then not only rooted in complex structure (or connectivity to use the language of CNT), an additional ingredient is the fact (already envisioned by Ludwig von Bertalanffy, the founder of General Systems Theory [11]) is that complex systems are open systems, i.e. ones that present strong interactions with their environment [12, $13]$.

\subsection{Emergent Properties, Cooperativity and Non-Linearity}

As stated previously the problem of emergence of complexity can be solved at several levels. One may say that the first one is in recognizing what parts of a system do 
together that they would not do by themselves, individually. Trivial examples abound: one protein is not a cell, one molecule of water is not liquid, one neuron alone does not compute and one person alone does not present social features. The question is thus related to the rise of collective phenomena: how do systems level properties arise from the properties of the parts connected (and the nature of the interactions between these)? This question may be answered at different organizational levels ranging from local interactions to more collective ones all the way up to the systems size [14]. In some cases, systems properties arise due to local interactions among constituents, without the presence of any external perturbation by means of a mechanism generalistically referred to as self-organization. In fact, emergent properties have been defined as the arising of novel and coherent structures, patterns and properties during the process of self-organization in complex systems [15]. Emergence is often non-trivial, there are systems for which emergence happens over several size and time scales thus forming topdown (or reverse) feedback in systems with emergent properties [16].

Emergent properties of complex systems thus depend on the presence of cooperativity which is the type of behavior where a number of apparently independent constituents of a system act collectively or synchronized. Cooperativity means that there is some mechanism of communication among the systems components. Information channels may vary drastically among different types of complex systems and so do the mechanisms of cooperativity. However, these often involve the flow of signals that while moving through the system activate (or deactivate) the dynamics. Systems which could not be characterized as complex also present cooperativity, but the patterns of influence are much simpler and the effects of such cooperation are predictable due to a linear character of the interactions. No synergistic phenomena is present.

Complex systems in the other hand, are quite often non-linear which means that cooperativity effects are not-additive, that signals are not linear and completely synchronous and that "effects" are not proportional to “causes”. Technically a non-linear system does not satisfy the so-called superposition principle that states that he net response at a given place and time caused by two or more stimuli is the sum of the responses which would have been caused by each stimulus individually. Complex systems thus present emergent properties that arise from cooperativity between their components; such cooperative phenomena is driven by non-linear interactions.

\subsection{Systems Theory and Network Approaches}

Contemporary systems theory view on complexity is that arises from what is called a complex adaptive system
(CAS). A CAS is a thus, a collection of individual constituents (or agents) with freedom to act in ways that are not always totally predictable (i.e. they are to a certain degree autonomous), and whose interactions are crosslinked in such a way that by means of interactions one agent may affect the conditions in which other agents act (i.e. they are cooperative). In brief, a complex system is a collection of autonomous agents cooperating (or anticooperating for the case) forming a single not-triviallyseparated unity. Hence, the reductionistic approach that has been the basis of most western science fails. One simply cannot decompose a system into a collection of quite simple subsystems, easy to study. Organization structure in complex systems is not something exogenous to their behavior, all the contrary, what has been called the architecture of complexity is an inherent part of the mechanisms of action in these systems and as such cannot be obviated from the analysis. This is precisely the purpose of systems thinking (see Figure 1).

Several formal frameworks exist for the analysis of systems, by far one of the most popular is the one derived from graph theory: the so-called network approach. Complex systems have been devised as graphs or networks in which the agents or constituents are nodes (or vertices) and interaction between them are links (or edges). Interactions in the network may be directed (in which there are clearly defined transmitters and receivers of the information flow across the network) or non-directed (in which agents interact via a symmetrical relationship). Examples of the first class are seniority networks in companies (e.g. I am not the boss of my boss), gene regulatory networks in biology (a target gene is notalways - a transcription factor of their own transcription factors), citation networks in scientific publication (the paper citing my paper cannot be cited on my paper),

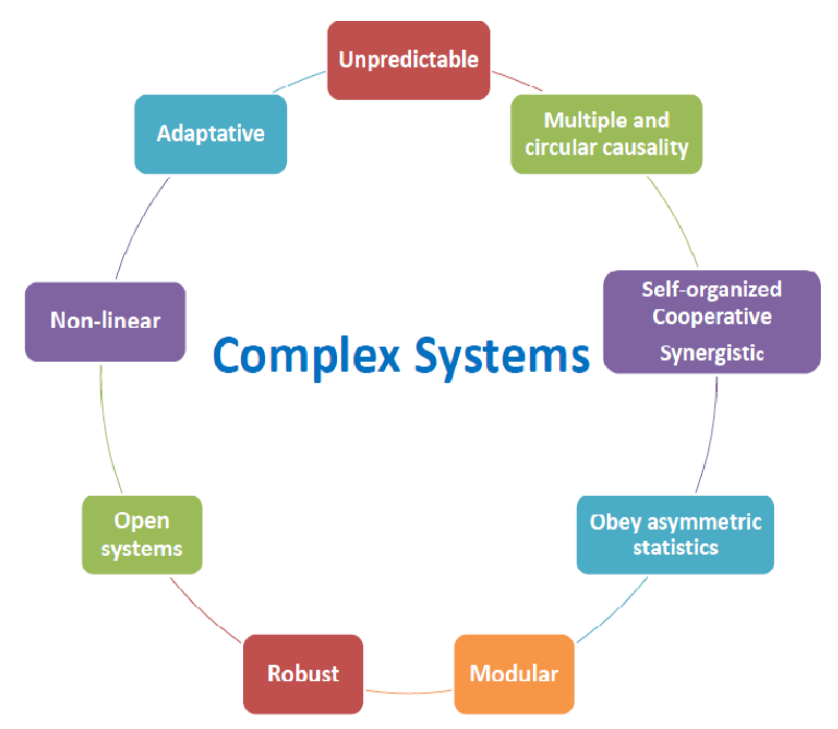

Figure 1. Main features of complex systems. 
etc. while example of the latter are social networks (in general, I am a friend of my friends), colleague and classmate networks and protein-interaction networks (if protein $\mathrm{A}$ is forming a complex with protein $\mathrm{B}$, then protein $B$ is also forming a complex with protein $A$ ). Network models have developed into very useful frameworks for the study of complex systems since they allow visualization of important features in cooperativity and other properties of complex systems, and since there is strong mathematical foundations of both the graph theoretical component and the probabilistic graphical models behind them. This second fact allows for a quantitative theory of complex networks that has resulted an extraordinary tool for the modeling of complex systems.

\section{Complex Features in Health Systems Design and Management}

For most practitioners, facing radical changes of contemporary health systems often represents a source of anxiety and frustration. Clinicians in particular, are accustomed to solve their problems based on a neat combination of rational deduction and personal experience $[17,18]$. While different in approach, this is also the way organization leadership and management (in particular in healthcare enterprizes) face their own challenges. In this traditional view, both human bodies [19] and organizations [3] are likened to simple machines which you repair and maintain by regularly oiling the parts and changing the defective gears. This simple, reductionistic (though highly effective in the past) view falls down when we recognize that health systems (both biological, social and organizational) are complex “devices" for which autonomy, independence or predictability conditions are seldom met $[19,20]$ The new paradigm of such “devices" is recognizing them as CASs [1]. As we recall, CAS present characteristic features: they are formed by a diverse, wide variety of elements that are able reshape themselves based on its previous history-i.e. they posses the ability to learn from experience-and these constituents are agents or autonomous beings. These agents act based on local knowledge on environmental conditions. A CAS hence, "...has a densely connected web of interacting agents, each operating from its own schema or local knowledge...” [21].

These characteristics are of course, present in health systems. For instance, due to the fact that every year billions of dollars are spent in biomedical research (both basic and applied) and drug-development, in the design of engineering devices to improve healthcare, in improving managerial strategies both in public health and in the private practice; and other changes, the health care environment is constantly changing, thus expressing the dynamic essence of CASs [22].

Health systems are also massively entangled. If we think about public health systems this is no wonder; a multitude of diverse elements compose such systems: physicians, nurses, epidemiologists, statisticians, chemists, biologists, but also engineers, physicists, mathematicians, accountants, lawyers, administrators, and even plumbers, janitors, carpenters and other types of handymen. All these different kinds of people, trained to work under different perspectives, with diverse working ethos and behavioral codes have to coexist and cooperate to shape the dynamics behind the function of health systems $[2,23]$.

Out of the complex relationships established by such diverse group of professionals there are critical points of confluence, say in the way decisions are made: In order to set a new surgical strategy, both the surgeon, the internist, the intensivist and the nursery team need to develop protocols of action. These protocols are nowadays developed within an integrative view taking different points of view and professional paradigms into account: Performing a faster (or easier or cheaper or in any form better) surgical procedure it is of no use if the patients die in the critical care unit. When planning to introduce a new diagnostic tool (say a new imaging technique) one must consult not only the needs and views of the radiologist or other physicians, but also the views of the biomedical engineer, the medical physicist or even the general services electrician of the hospital: A new PET scanner is of no use if there are strong concerns about safety or powermanagement performance. Said protocols would not be possible without a strong cooperative environment within all different players (or agents) and are thus an emergent property of health systems.

\subsection{Network and Community Structure in Health Systems}

When we consider examples as the ones above, one may wonder how is it that some current health systems actually work without taking into account the important role of complexity [24]. The answer is that most of this systems work under a lot of pressure and conflict [25], which may be largely diminished when planning health systems design and performance under the paradigm of complex science [22]. For instance, one may recognize that the traditional view of health systems (and other enterprizes) as mostly vertical hierarchical organigrams is one that, while may (sometimes) work in terms of authority, is not functional in real life [26]. For the sake of decision-making it is quite common that more than one information path needs to be taken into consideration [27-30].

Let us consider a hypothetical case we may recall a couple of times in this paper to illustrate the role of complexity in health care in a figurative way: we will call this scenario the one of Neurosurgery and the janitor. As the 
name implies, it involves two apparently disparate positions in healthcare systems. Let us imagine a situation (for many service heads in large hospital will not be hard to imagine this) in which the person in charge to clean the surgical room devoted to neurosurgery in a large hospital is not present (or its job is not done in time or in a proper way). When the surgical staff gets into the surgical room, they notice it is not properly cleansed. They report this to the neurosurgeon in charge. He/She in turn reports this to the head of the neurosurgery service. In turn the service head talks with the medical subdirector. The medical subdirector talks to the general services director, who in turn talks with the head of hospital maintenance. He/She talks to the cleaning-tasks manager to ask for someone to please go clean the surgical room. Now imagine this happens early in the morning when likely not all the involved authorities are present and that this particular surgery is an emergency procedure. Those for something so simple and common as a failure in janitorial services a human life is at stake because of the delay. This brings up not only management inconvenience but also bioethical (and likely even legal) issues.

One may recall that even in large hospitals is not common to have more (highly specialized and equipped, thus expensive) surgical rooms for neurosurgery than the ones actually in use. Hence, it is not likely that one can just move the patient to the next (well cleansed) surgical room. This situation although hypothetic is useful to explain a feature of complex systems: they are complex irreducible which means that one cannot take a single part (even one that seems of little importance at first glance) without changing (sometimes in quite dramatic ways) the dynamics and functionalities of the whole system.

Interestingly enough, it is a common practice for the heads of surgical services to have replacement for their surgeons, anesthetists, nurses in case of eventualities. However, none to date is currently aware of situations like the missing janitor! This is so since the actual administrative organization is extremely vertical and fragmented so that separate branches do not touch themselves (say neurosurgery and janitorial services). As an example, in Figure 2 we present the organic structure of an actual emergency and trauma reference hospital. Boxes are color-coded: blue is medical area, orange is management/administration and red is Director General. This linear structure is in stark contrast with the (more realistic) network structured model in which communication links are established between different players disregarding the traditional line of authority [31]. In Figure 3 we may compare the hierarchical complexity of an organigram and network community structure (network

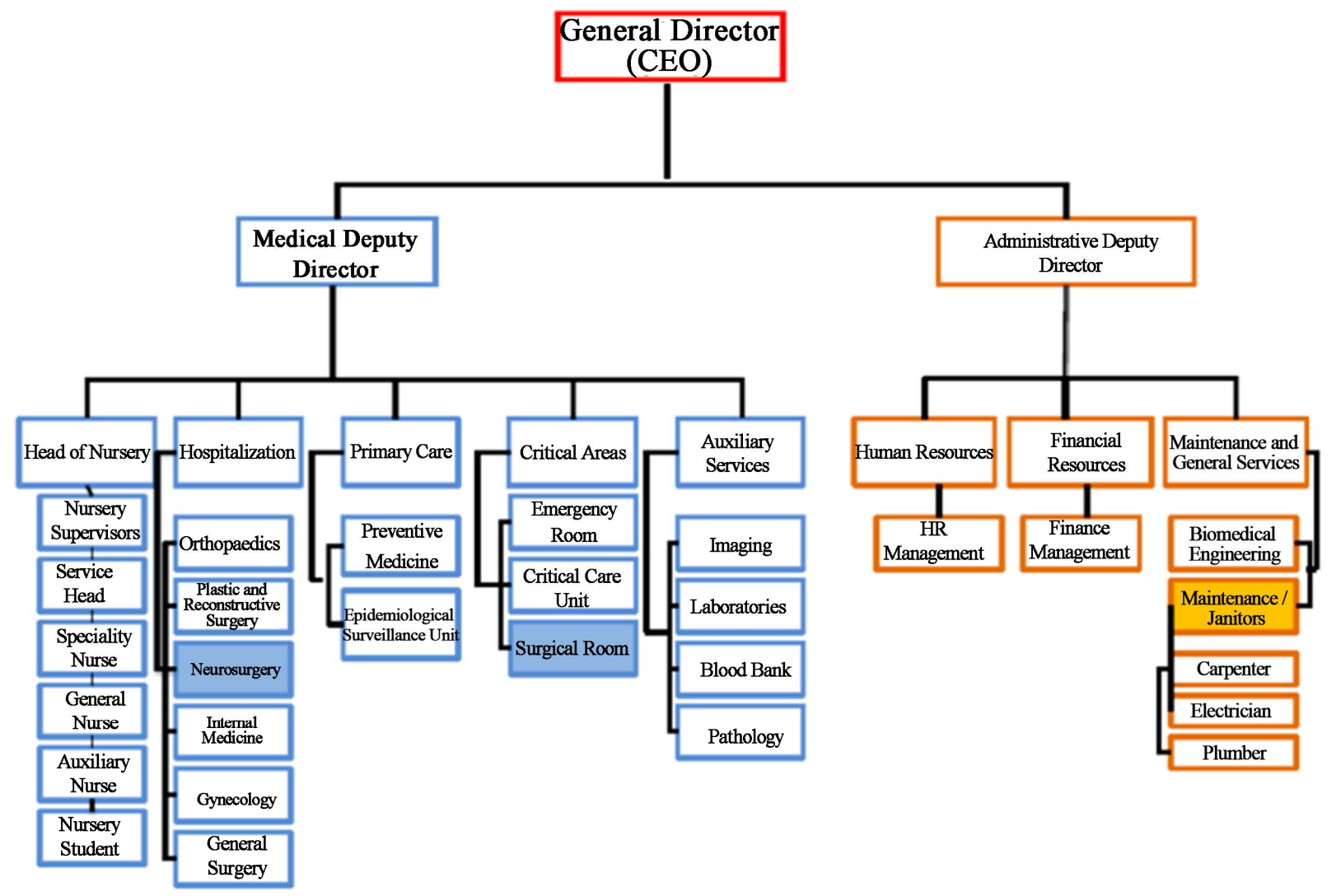

Figure 2. Organic structure of an actual emergency and trauma reference hospital. 
depicted just for illustrative purposes, without real-life counterpart yet) for an actual healthcare institution (Same as in Figure 2, see Figure 3 panels a and b, respectively; same color-codes). One can notice that while in Figure 3(a) (same as Figure 2) there are not interdepartmental connection links, in Figure 3(b) separate departments are actually connected. In Figure 3(c) we can see the same network structure as in Figure 3(b). Nodes (Agents) are size-coded according with their so-called connectivity degree, i.e. big nodes are people that actually interact with a lot of people, these are key individuals within an organization.

\subsection{Cooperative Dynamics in Health Systems}

The above discussion also serves to illustrate the role that cooperative dynamics [23] may play in healthcare institutions. The competitive nature of human interaction frequently presents a challenge for cooperation, hence for the good performance of health systems. The common differences in views: surgeons versus internists, clinicians versus administrative teams, etc. may present challenges way beyond the anecdotic [32]. Understanding the social dynamics of these different groups in terms given by the theory of complex social systems [33] may help health policy designers and operative authorities to improve the strategies to enhance cooperation and diminish conflict. An interesting case study for nursing management is given in reference [34].

The dynamics of cooperation need to be involved in issues such as the one in leadership and decision making [35]. This cooperation in health system must go well beyond the one attained by a group of colleagues within an institution. Healthcare systems functioning result from the network of constituents at different hierarchic and organizational levels: from hospital, clinics and nursing homes to rehab units to patients' homes. All these components communicate with the others in a highly nonlinear fashion and in different scales [36]. Failure in cooperation within such units: a hospital declining admission to a patient from a given clinic or a patient's relative failing to administer drugs and medications to the patient will result in disruption of the intended health care.

\subsection{Non-Linearity in Health Systems Response}

Since we have already considered how emergent properties arise from the interaction of synergistic agents in a complex environment, and as already cartooned in the illustration about Neurosurgery and the janitor, health systems are also complex in the fact that their responses are strongly non-linear: this is the size of the response is not proportional to the size of the stimulus. One may think that a performance failure in a non-medical office (the janitor) may have almost no consequences on the performance (and the results) of a well trained body of surgical specialists (the neurosurgeons, anesthetists, intensive care specialists and so on). Reflecting about the effect that bacterial infections may have in the central nervous system of an immunologically suppressed surgical patient will convince us otherwise. "Small" stimuli may often produce tremendous effects. Even more dramatic amplifications may occur at the level of public health problems like pandemic diseases.

\subsection{Health Systems Robustness and Adaptability}

Due to their inherent network structure and strong connectivity, complex systems may be extremely robust to failure of a number of their non-critical constituents. This is so since, in stark contrast with extremely rigid architectures given by traditional hierarchic structures, networked systems (assumed as such) are flexible to a certain extent. This fact along with the strong communication capacities of agents within the network. Made possible for the complex systems to be adaptable, hence robust.

Let us revisit the case of the Neurosurgery and the janitor but now let us consider that instead of the rigid hierarchic structure already mentioned, the hospital is organized as a complex system, in particular under the network paradigm. Instead of working under the onetask-one-person paradigm, complex networks often work in modules, i.e. groups of individual agents that perform related tasks together. Modular structure is extremely common in natural complex systems. For instance, signaling pathways within the cell function by the action of several different molecules (a module) that may act in a variety of arrangements in such a way that if one of them is missing (say by a disease or a temporal disequilibrium) the signaling task is still possible to perform (although maybe in a slightly less efficient way).

In fact, in a rather counter-intuitive fashion, some damaged complex networks actually rearrange themselves to regain efficiency by further damage [37].

Instead of having the man-that-cleans-neurosurgicalroom-4, you have a team of janitors that altogether clean the surgical rooms; instead of having a set of individual heads of services you have a (real, not in paper) board of surgical experts (likely each one in charge of one service still, due to specialization) that is extremely well communicated and cooperative with each other; last but not least, the medical director, the administrative director and the general services director are not strange to each other but are communicated (linked to each other in the network). What will happen then: A janitor is missing, the team of janitors easily notices and reorganize itself so as to (with some extra work and maybe a little overtime) have all the needed surgical rooms well cleansed. In the 


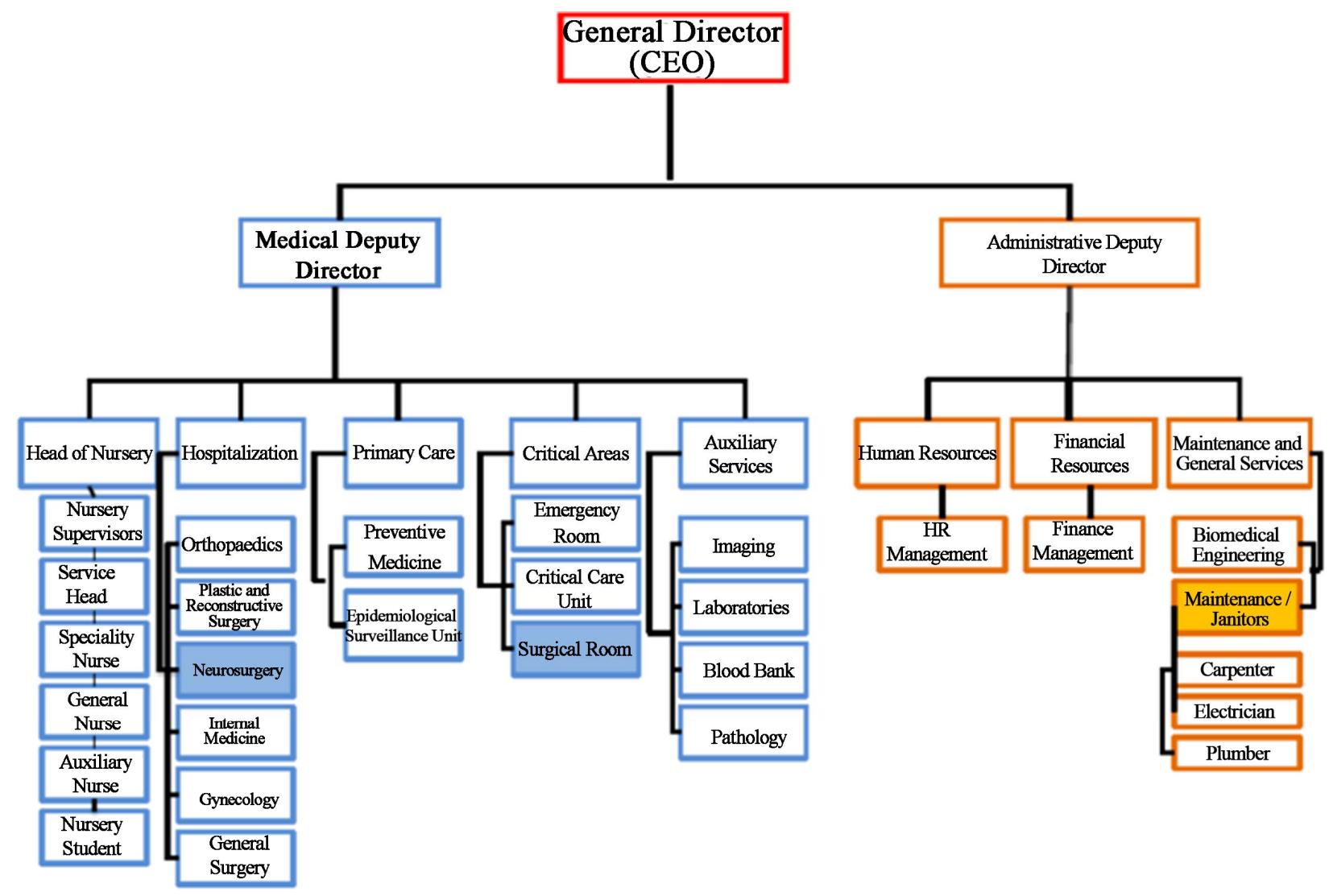

(a)

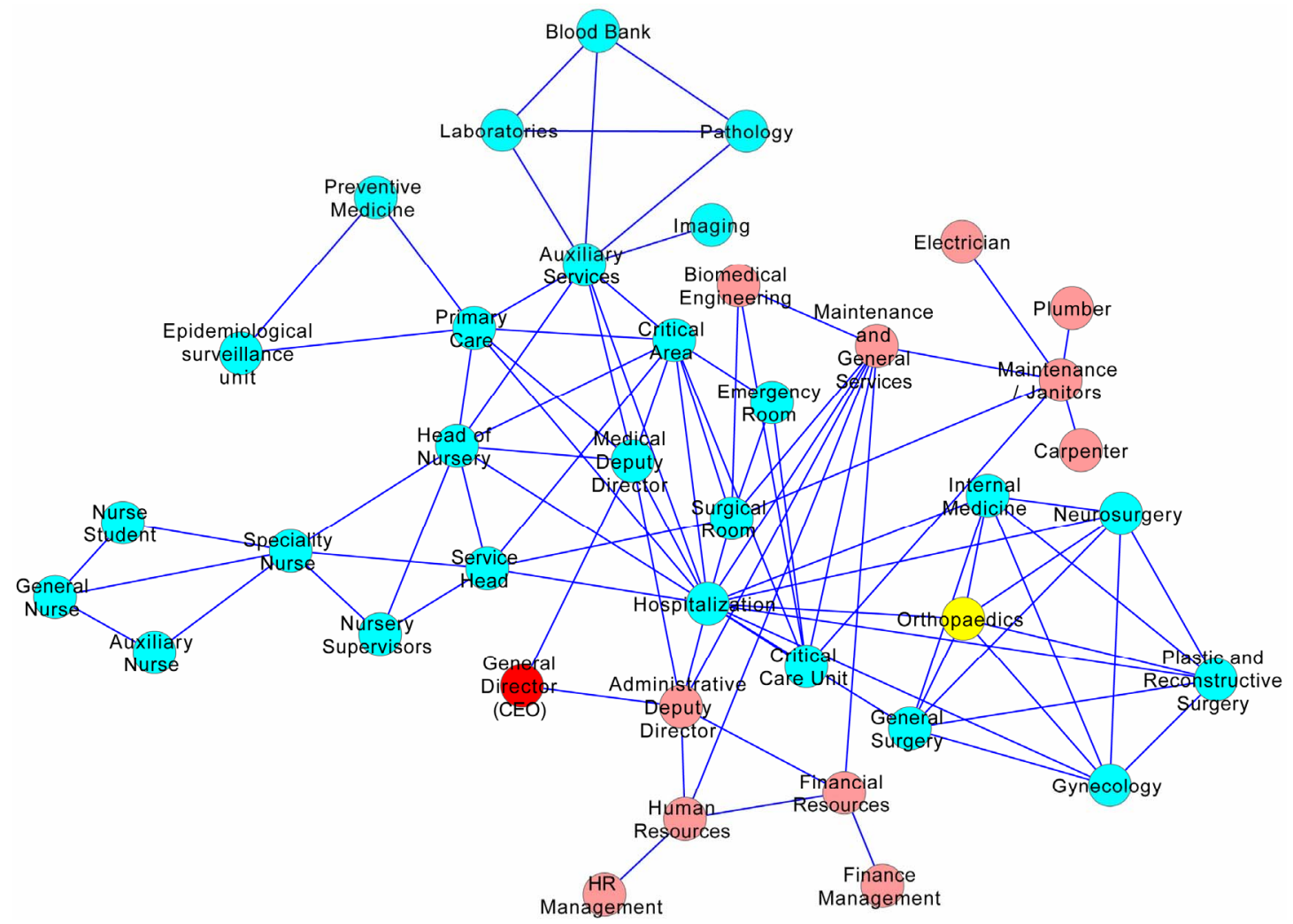

(b) 


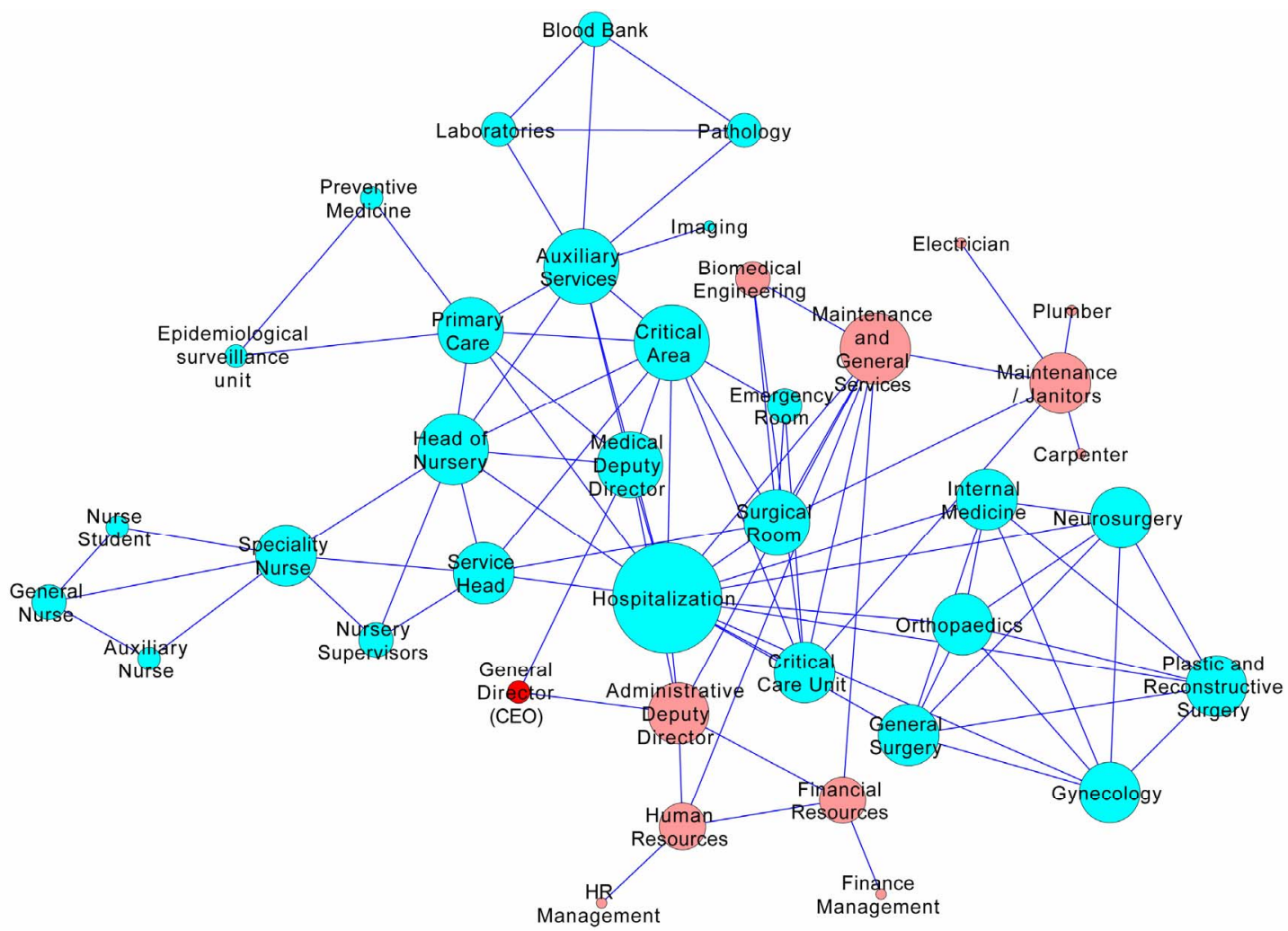

(c)

Figure 3. Organic and Network structure of an actual emergency and trauma reference hospital. Panel A-same as Figure 2-presents the traditional organigram view. Panel B presents an illustrative network depiction of the same hospital. Panel C is same as panel B but nodes are size-coded according with the number of functional links. One can see that there is a diversity of communication profiles which is actually identifiable in contrast with the organigram.

meantime the board of service heads (even if it is not complete at that moment) decides which surgery-scheduled patients are the more urgent and promptly rearrange their room-schedule so as to have the more urgent patients go into the first available rooms. In the end, the group of directors is already aware of the circumstances and may plan ahead for future re-design and re-engineering of the hospital procedures at several levels. We may call this the complex approach to the Neurosurgery and the janitor problem. Of course, one may argue that this is more or less what many (but not all as some health care providers may witness) hospitals and health care organization do based in their own experience. However, such adjustments often happen over-the-norm and are not part of the original organizational design and structure of the institutions, often rely on the good will of the people involved and-what is more critical-often generate stress and anxiety in the personnel involved due to unawareness.

\subsection{Hierarchical Complexity and Health Systems Management}

The study of complex systems has shown us that knowl- edge about the architecture of complexity is just a first step towards the understanding of the underlying dynamics and its control. Recognizing health systems as complex systems is just the beginning. For instance, contrary to what traditional policy makers sustain, having more rules and stronger normativity may cause health systems to actually perform worst than before. It has even been stated that "...As more regulations are created to control the behavior of a complex system, the more the system may deviate from a desired outcome..." [36] based on cases such as the redesign of the Australian healthcare structure [38]. The study of Sturmberger et al. [38], actually discourages the use of normative strategies such as the so-called disease protocols (i.e. the fact that every specific disease - or set of diseases - must be treated according with a pre-established set of context-independent rules), or the use of financial levers since their mid to long term effects are unpredictable and often negative. The same has been observed in other cases such as payfor-performance and similar strategies supposedly used to improve hospital performance; and in the use of clinical practice guidelines: there is evidence that they have not resulted in lowering mortality, insurance costs, or reducing socioeconomic disparities on the treatment of 
common complex diseases (see [36] and references therein). In brief, they have not solve the problems they were aimed at, and in some instances they even worsen the situation $[39,40]$.

This is so, since in complex systems (as we have discussed health systems are) one cannot get rid-out of a certain degree of unpredictability, which inherent to their functioning and which should be accounted for when designing strategies [41,42]. Contrary to traditional views, unpredictability do not necessarily dictate uncontrollability but the control mechanisms of complex systems are different since they must account for redundancy, flexibility and enhanced communication, which are natural features of complex systems $[43,44]$. If we recall what we called the complex approach to the Neurosurgery and the janitor problem we can see that these three elements are indeed central to the control of the system.

\section{The Six-Sigma $(6 \sigma)$ and Other Quantitative Approaches to Health Systems}

Quality management is a major strategic issue in healthcare organizations. Based on proposals made to improve health quality, multiple valid and reliable measurements have been developed which focus on process and the results. These metrics are service level, service cost, customer satisfaction, and clinical excellence. While these metrics are applicable in healthcare organizations, they are also very difficult to apply in a health care setting. Amongst these we can mention the International Quality Standards ISO 9000 series, the European Quality Award, INK-award, the MBNQA, the Lean strategy, the Theory of Constraints approach, the Total Quality Management and the Six-Sigma Approach. The latter is the most comprehensive and the closest in spirit to complex systems modelisation [45].

To date, there is not a general framework to study, design, implement and optimize the performance of health systems (or, for the case, of any other complex systems management strategies). However, a methodology that it is somehow closer in spirit to complex systems is the so-called Six-sigma ( $6 \sigma$ ) approach. Six sigma is an organized systematic method for strategic process improvement based on statistical and scientific methodology to achieve drastic reductions in failures of processes. In order to quantify the performance of a given process a Six Sigma project starts by defining and implementing relevant measures and metrics, the so-called Critical. This powerful management strategy combines improved metrics and cook-book methodology strengthening a company's market position, Statistical Process Control and enhancing the financial impact to the bottom-line [46]. The Greek letter sigma ( $\sigma$ ) is used in statistics to denote the standard deviation (a measure of dispersion of data from the mean value). The higher the value of $\sigma$ and, consequently, the lower the standard deviation, the process is better, more accurate and less variable. In statistics the value of $6 \sigma$ means 3.4 defects per million [45].

Six-sigma recognizes the cooperative and synergistic nature of organizational systems; this way, instead of focusing in the execution or performance of individual tasks and their control and optimization, it concentrates on global results (in particular in establishing a higher bound on the level of operational errors permitted overall). Under the six-sigma paradigm, organizational units are no longer isolated components or gears in a machinery but rather they are conformed (one may say self-organized) in highly connected groups-termed belts and distinguished by colors according to function (Figure 4) within the organizational structure-of people (agents) that may in some cases recognized as modular units within the so-called matrix which represents the organizational network of the system.

The six-sigma approach relies on a looped feedback structure to perform processes design and re-engineering. In this scheme, systems are subject to the so called DMAIC optimization, that is repeated until the desired performance is attained (see Figure 5). Steps in this DMAIC optimization are not independent but cooperative. They are not necessarily sequential since it is possible, for instance, that while implementing an improvement strategy (I), new points-for-improvement are discovered and the diagnose (definition) is altered (D).

\section{How to Improve Health Systems Design and Management by Using the Theory of Complexity?}

Once we know how health systems behave as complex systems, and after having been considered the way that complex systems are structured, how they function and even how they may be possibly controlled, a few points should be clear now:

1) Health systems must be recognized as complex systems and their design strategies should be based on such premise (for an illustrative set of examples of applicability of the complexity paradigm refer to Figure 6).

2) As such, health systems organic structures should be built based in the modular network paradigm instead of the traditional vertical hierarchic structure of the past.

3) In order to do so, the system should be design by means of functional decomposition strategies, i.e. by recognizing the modular structure of the network based on mechanistic approaches and not only in organizational levels.

4) This should be reflected by open mechanisms of communication among agents in such networks as well 


\section{Six-sigma levels of expertise}

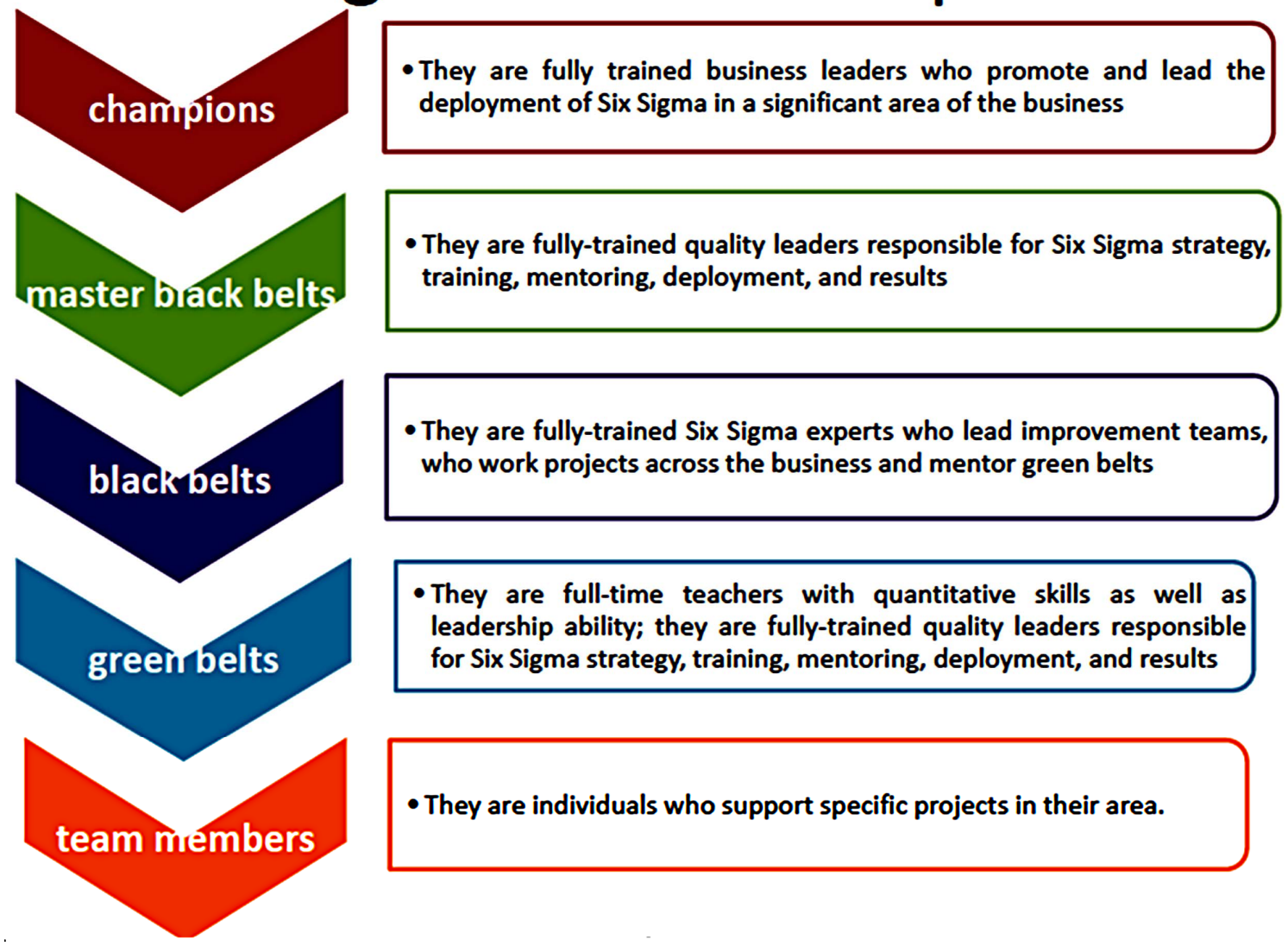

Figure 4. The modular structure of different belts under six-sigma.

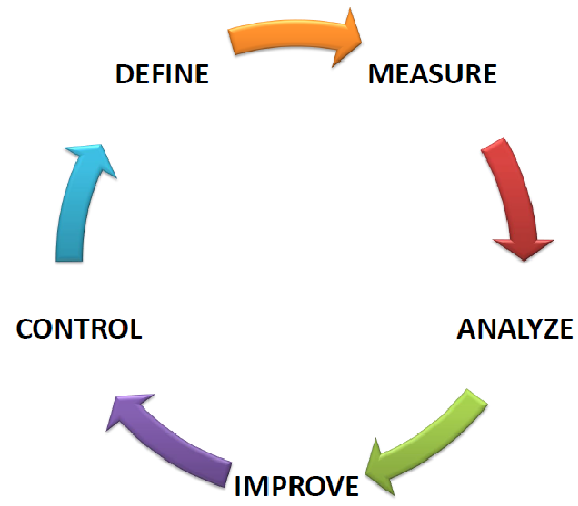

Figure 5. The DMAIC optimization cycle.

as by a flexible architecture that allow for robustness, adaptability and evolvability of the systems as time goes by (Figure 7).

5) Since health systems are complex adaptive systems, organizational changes must be driven by the dynamics of the complex system itself and not by any personal agendas of the involved agents, not even those of the ones in charge.

6) Problems arising might be of three kinds: overuse, underuse and misuse (see Figure 8). Instead of treating them separately they must be recognized as a single synergistic source of conflict.

7) In order to actually develop such strategies, a new culture should be adopt by all the healthcare personnel, that of cooperativity and synergy instead of that of individualistic performance. In this culture, the value of the team work (understood as a network module) is fundamental.

\section{Conclusions}

We have considered many instances for which health systems at different levels present complex behavior, characteristic of Complex Adaptive Systems. These features of complexity represent a challenge for those in charge of the design and implementation of public health policies, as well as private and social strategies for the appropriated performance of health systems. As it is, most of these issues required new ways to implement the logistics that are in a close spirit of traditional optimization schemes in operations research. However, most of them have been treated at the light of some kind of strategic planning philosophy, i.e. strongly reliant on heuristics 

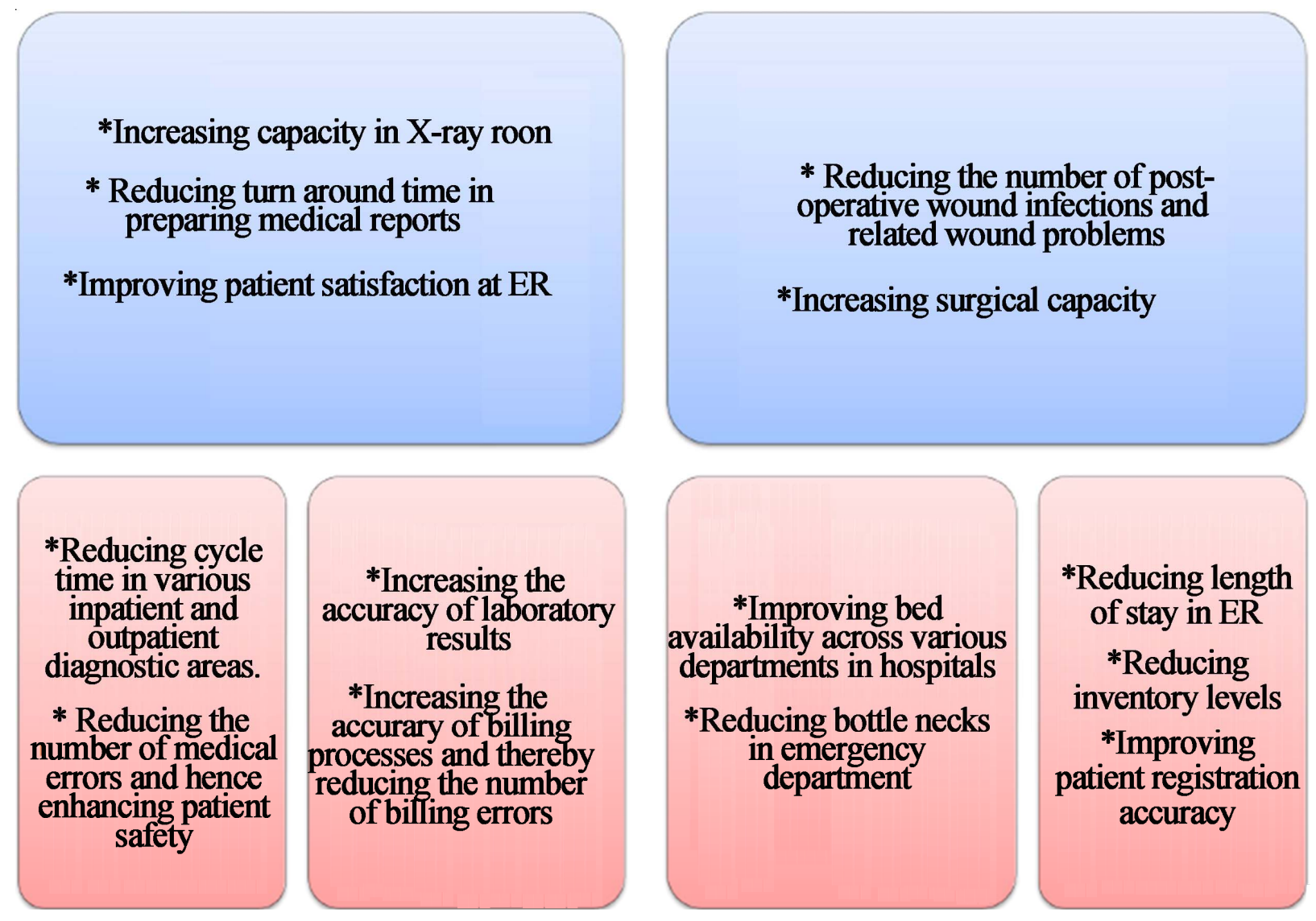

Figure 6. Potential areas in Healthcare Industry where six sigma could be applied.

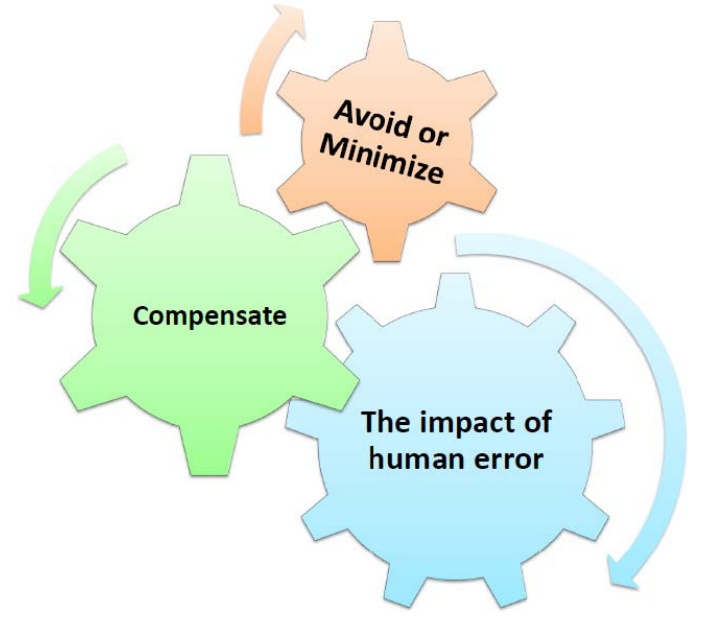

Figure 7. Within the six-sigma approach, error is controlled by two mechanisms based on cooperativity and self-organization: error minimization (mostly at the individual unit level) and compensation based in synergistic actions of groups.

and the wealth of personal experience of the person (or set of persons) in charge. In a world that is becoming more and more complex this strategy is destined to fail at one point or another.

A failure that may be especially dramatic within the setting of healthcare enterprizes due to the role in the social, economic and humanistic development of socie- ties. For this reason we propose the study of health systems at the light of the sciences of complexity, and the implementation and control of their performance in terms of quantitative quality measures strongly based in modern statistics and non-linear optimization. The so-called six-sigma approach is just one of the managerial paradigms that may be implemented with this goal in mind. 
(a)

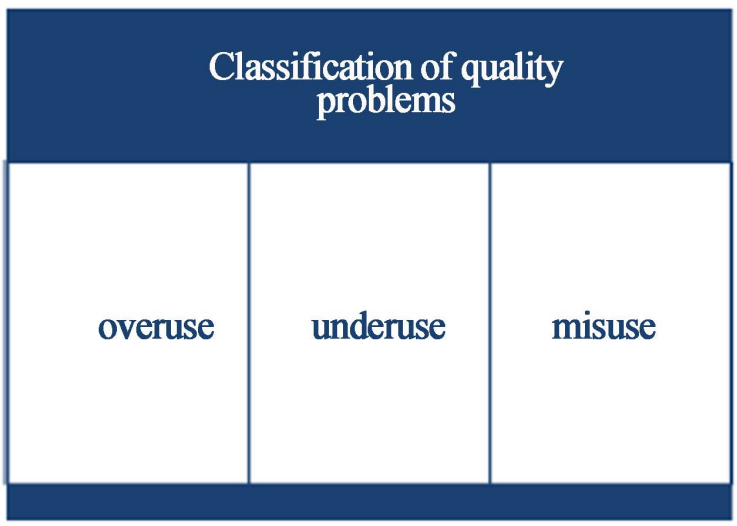

(c)

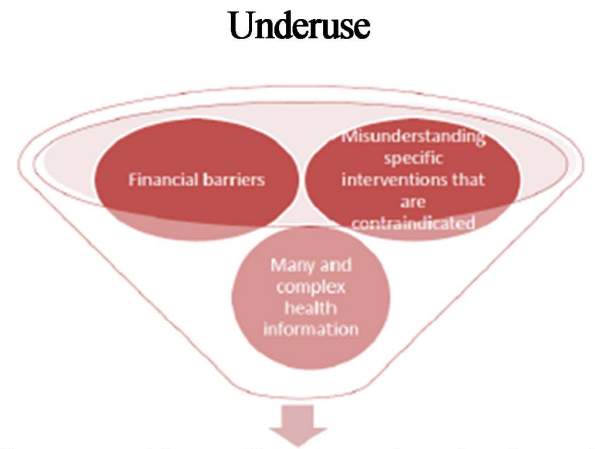

Failing to provide an effective service when it would have produced favorable outcome constitutes underuse (b) Overuse

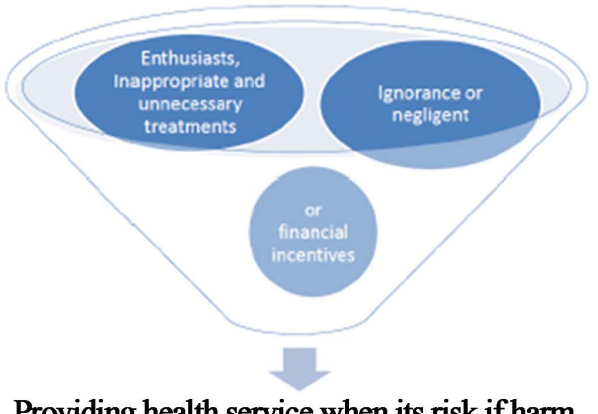

Providing health service when its risk if harm exceeds its potential benefit constitutes overuse

(d)

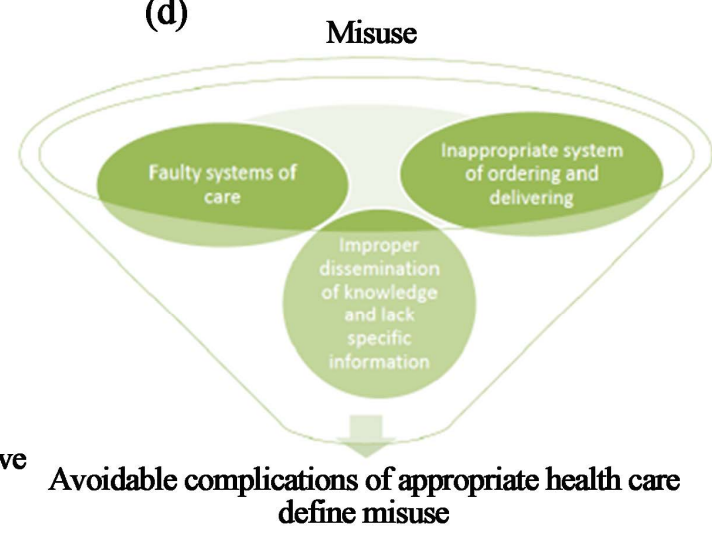

Figure 8. Panel A Classification of quality problems in health systems performance according with the six-sigma paradigm. Panel B Overuse, Panel C Underuse, Panel C Misuse.

Much work has still to be done however to reach a complete understanding of complexity in health systems at a level that allow for precise control and optimization of performance, but we believe complex systems theory and its methods may well lie a foundation for it.

\section{REFERENCES}

[1] P. E. Plsek and T. Greenhalgh, "The Challenge of Complexity in Health Care,” British Medical Journal, Vol. 323, No. 7313, 2001, pp. 625-628, doi:10.1136/bmj.323.7313.625

[2] P. Anderson, "Complexity Theory and Organization Science,” Organization Science, Vol. 10, No. 3, 1999, pp. 216-232. doi:10.1287/orsc.10.3.216

[3] B. J. Zimmerman, C. Lindberg and P. E. Plsek, "Edgeware: Complexity Resources for Healthcare Leaders," VHA Publishing, Irving, 1998.

[4] L. B. Smith and E. Thelen, "Development as a Dynamic System,” Trends in Cognitive Sciences, Vol. 7, No. 8, 2003, pp. 343-348. doi:10.1016/S1364-6613(03)00156-6

[5] H. Maturana and F. Varela, “Autopoiesis and Cognition,” Reidel, Dordrecht, 1980. doi:10.1007/978-94-009-8947-4

[6] F. Baumgartner and B. Jones, "Positive and Negative Feedback in Politics in Policy Dynamics," In: F. Baumgartner and F. B. Jones, Eds., University of Chicago Press,
Chicago, 2002.

[7] T. Schelling, "Micromotives and Macrobehavior," Norton Publishing, New York, 1978.

[8] T. Schelling, "Dynamic Models of Segregation,” Journal of Mathematical Sociology, Vol. 1, No. 2, 1971, pp. 119132. doi:10.1080/0022250X.1971.9989794

[9] H. Simon, “The Architecture of Complexity," Proceedings of the American Philosophical Society, Vol. 106, No. 6, 1962, pp. 467-482.

[10] R. May, "Simple Mathematical Models with Very Complicated Dynamics,” Nature, Vol. 261, No. 5560, 1976, pp. 459-467. doi:10.1038/261459a0

[11] L. V. Bertalanffy, “General Systems Theory,” George Braziller, New York, 1968.

[12] D. Bernstein, "Feedback Control: An Invisible Thread in the History of Technology," IEEE Control Systems Magazine, Vol. 22, No. 2, 2002, pp. 53-68. doi:10.1109/37.993315

[13] P. Erdi, "Complexity Explained, Springer Complexity Series,” Springer-Verlag, Berlin, 2008.

[14] J. P. Crutchfield and M. Mitchell, "The Evolution of Emergent Computation," Proceedings of the National Academy of Sciences of the USA., Vol. 92, No. 23, 1995, pp. 10742-10746. doi:10.1073/pnas.92.23.10742

[15] J. Goldstein, "Emergence as a Construct: History and 
Issues,” Emergence: Complexity and Organization, Vol. 1, No. 1, 1999, pp. 49-72.

[16] A. Korotayev, A. Malkov and D. Khaltourina, "Introduction to Social Macrodynamics: Compact Macromodels of the World System Growth,” URSS, Moscow, 2006.

[17] C. Brown, "The Application of Complex Adaptive Systems Theory to Clinical Practice in Rehabilitation," Disability and Rehabilitation, Vol. 28, No. 9, 2006, pp. 587593. doi:10.1080/00222930500219175

[18] H. Benson, "Chaos and Complexity: Applications for Healthcare Quality and Patient Safety," Journal for Healthcare Quality, Vol. 27, No. 5, 2005, pp. 4-10. doi:10.1111/j.1945-1474.2005.tb00571.x

[19] A. L. Goldberger, "Nonlinear Dynamics for Clinicians: Chaos Theory, Fractals, and Complexity at the Bedside," Lancet, Vol. 347, No. 9011, 1996, pp. 1312-1314. doi:10.1016/S0140-6736(96)90948-4

[20] H. R. Priesmeyer, L. F.Sharp, L. Wammack and J. D. Mabrey, "Chaos Theory and Clinical Pathways: A Practical Application," Quality Management in Health Care, Vol. 4, No. 4, 1996, pp. 63-72.

[21] J. W. Begun, B. Zimmerman and K. Dooley, "Health Care Organizations as Complex Adaptive Systems,” In: S. M. Mick and M. Wyttenbach, Eds., Advances in Health Care Organization Theory, Jossey-Bass, San Francisco, 2003, pp. 253-288.

[22] M. Arndt and B. Bigelow, "Commentary: The Potential of Chaos Theory and Complexity Theory for Health Services Management," Health Care Management Review, Vol. 25, No. 1, 2000, pp. 35-38. doi:10.1097/00004010-200001000-00004

[23] R. Axelrod, “The Evolution of Cooperation,” Basic Books, New York, 1984.

[24] G. J. Bazzolli, S. M. Shortell, N. Dubbs, C. Chan and P. Kralovec, "A Taxonomy of Health Networks and Systems: Bringing Order out of Chaos," Health Services Research, Vol. 33, No. 6, 1999, pp. 1683-1717.

[25] G. T. Savage and A. M. "Roboski, "Integration as Networks and Systems: A Strategic Stakeholder Analysis,” Advances in Health Care Management, Vol. 2, Elsevier Science, London, 2001, pp. 37-62

[26] R. Cross and A. Parker, "The Hidden Power of Social Networks," Harvard Business School Press, Boston, 2004.

[27] R. Agranoff and M. McGuire, "Managing in Network Settings,” Policy Studies Review, Vol. 16, No. 1, 1999, pp. 18-41. doi:10.1111/j.1541-1338.1999.tb00839.x

[28] J. Anderson, "Evaluation in Health Informatics: Social Network Analysis," Computers in Biology and Medicine, Vol. 32, No. 3, 2002, pp. 179-193. doi:10.1016/S0010-4825(02)00014-8

[29] S. P. Borgatti and P. C. Foster, "The Network Paradigm in Organizational Research: A Review and Typology," Journal of Management, Vol. 29, No. 6, 2003, pp. 9911013.

[30] S. P. Borgatti and J. L. Molina, "Ethical and Strategic Issues in Organizational Social Network Analysis," Journal of Applied Behavioral Science, Vol. 39, No. 3,
2003, pp. 337-349. doi:10.1177/0021886303258111

[31] P. S. Dodds, D. J. Watts and C. F. Sabel, "Information Exchange and the Robustness of Organizational Networks," Proceedings of the National Academy of Sciences, Vol. 100, No. 21, 2003, pp. 12516-12521. doi:10.1073/pnas.1534702100

[32] T. Wilson, T. Holt and T. Greenhalgh, "Complexity Science: Complexity and Clinical Care,” British Medical Journal, Vol. 323, No. 7314, 2001, pp. 685-688. doi:10.1136/bmj.323.7314.685

[33] R. Axelrod and M. D. Cohen, "Harnessing Complexity: Organizational Implications of a Scientific Frontier,” Free Press, New York, 1999. doi:10.5812/jhs.4623

[34] J. W. Begun and K. R. White, "The Profession of Nursing as a Complex Adaptive System: Strategies for Change,” In: J. J. Kronenfeld, Ed., Research in the Sociology of Health Care, JAI Press, Lexington, 1999. pp.189-203

[35] A. Baghbanian, G. Torkfar and Y. Baghbanian, "Decision-Making in Australia's Healthcare System and Insights from Complex Adaptive Systems Theory," Health Scope, Vol. 1, No. 1, 2012, pp. 29-38. http://dx.doi.org/10.5812/jhs.4623

[36] L. A. Lipsitz, "Understanding Health Care as a Complex System: The Foundation for Unintended Consequences," The Journal of the American Medical Association (JAMA), Vol. 308, No. 3, 2012, pp. 243-244. doi:10.1001/jama.2012.7551

[37] A. E. Motter, N. Gulbahce, E. Almaas and A. L. Barabási, "Predicting Synthetic Rescues in Metabolic Networks," Molecular Systems Biology, Vol. 4, 2008, Article No. 168. doi:10.1038/msb.2008.1

[38] J. P Sturmberg, D. M. O’Halloran and C. M. Martin, "Understanding Health System Reform-A Complex Adaptive Systems Perspective," Journal of Evaluation in Clinical Practice, Vol. 18, No. 1, 2012, pp. 202-208. doi:10.1111/j.1365-2753.2011.01792.x

[39] A. Shiell, P. Hawe and L. Gold, "Complex Interventions or Complex Systems? Implications for Health Economic Evaluation,” British Medical Journal, Vol. 336, No. 7656, 2008, pp. 1281-1283. doi:10.1136/bmj.39569.510521.AD

[40] C. Lessard, "Complexity and Reflexivity: Two Important Issues for Economic Evaluation in Health Care,” Social Science \& Medicine, Vol. 64, No. 8, 2007, pp. 1754-1765. doi:10.1016/j.socscimed.2006.12.006

[41] R. A. Anderson, and R. R. McDaniel Jr., "Managing Health Care Organizations: Where Professionalism Meets Complexity Science," Health Care Management Review, Vol. 25, No. 1, 2000, pp. 83-92. doi:10.1097/00004010-200001000-00010

[42] J. Paley, "The Appropriation of Complexity Theory in Health Care,” Journal of Health Services Research \& Policy, Vol. 15, No. 1, 2010, pp. 59-61. doi:10.1258/jhsrp.2009.009072

[43] T. G. Kannampallil, G. F. Schauer, T. Cohen and V. L. Patel, "Considering Complexity in Healthcare Systems," Journal of Biomedical Informatics, Vol. 44, No. 6, 2011, pp. 943-947. doi:10.1016/j.jbi.2011.06.006

[44] K. Resnicow and S. E. Page, "Embracing Chaos and 
Complexity: A Quantum Change for Public Health,” American Journal of Public Health, Vol. 98, No. 8, 2008, pp. 1382-1389. doi:10.2105/AJPH.2007.129460

[45] J. van den Heuvel, "The Effectiveness of ISO 9001 and Six Sigma in Healthcare,” Beaumont Quality Publications,
Beaumont, 2006.

[46] H. Bevan, N. Westwood, R. Crowe and M. O’Connor, "Lean Six Sigma: Some Basic Concepts," NHS Institute for Innovation and Improvement, Nottingham, 2007. 